\title{
Ionic Disorders in Malaria and Dengue Co-Infection
}

\author{
Fabienne M. Soudre ${ }^{1,7}$, Arnaud Kouraogo ${ }^{3}$, Alice T.C.R. Kiba ${ }^{4,7}$, Raoul Karfo ${ }^{3,7}$, Thierry Guiguemde ${ }^{2,7}$, Bibata Kabore, \\ Elie Kabre ${ }^{6,7}$, Jean Sakande ${ }^{3,7}$ \\ ${ }^{1}$ Biochemistry Unit, Pediatric University Teaching Hospital Charles de Gaulle, Ouagadougou, Burkina Faso \\ ${ }^{2}$ Parasitology Unit, Pediatric University Teaching Hospital Charles de Gaulle, Ouagadougou, Burkina Faso \\ ${ }^{3}$ Biochemistry Department, University Teaching Hospital Yalgado Ouédraogo, Ouagadougou, Burkina Faso \\ ${ }^{4}$ Biochemistry Service, University Teaching Hospital of Tengandogo, Ouagadougou, Burkina Faso \\ ${ }^{5}$ Immunology-Serology Unit, Pediatric University Teaching Hospital Charles de Gaulle, Ouagadougou, Burkina Faso \\ ${ }^{6}$ National Laboratory of Public Health, Ouagadougou, Burkina Faso \\ ${ }^{7}$ Training and Research Unit in Health Sciences, University Joseph Ki-Zerbo, Ouagadougou, Burkina Faso \\ Correspondence: Fabienne M. Soudre, Pediatric University Teaching Hospital Charles de Gaulle, Ouagadougou, \\ Burkina Faso.
}

Received: October 3, 2021 Accepted: November 1, 2021 Online Published: November 3, 2021

doi:10.5539/ijc.v14n1p1 URL: https://doi.org/10.5539/ijc.v14n1p1

\begin{abstract}
Introduction: The aim of this study was to investigate ionic disorders in malaria and dengue co-infection at Ouagadougou, Burkina Faso.

Material and methods: This is a descriptive cross-sectional study with retrospective data collection, carried out in the laboratory of the Pediatric University Hospital Charles de Gaulle in Ouagadougou, Burkina Faso, from January $1^{\text {st }}, 2017$ to December $31^{\text {st }}, 2019$. The study was on patients who performed a thick blood drop/smear, dengue serology and blood ionogram.

Results: On 1405 cases included in the study, 102 patients (7.26\%) were confirmed of malaria. Dengue serology was positive in 235 patients $(16.72 \%)$. The frequency of co-infection was $1.14 \%(\mathrm{n}=16)$. The mean age of the patients was 9.93 years and the age group of 0 to 15 years represented $78.93 \%$ of the cases. There was a male predominance with a sex ratio $(\mathrm{M} / \mathrm{F})$ of 2.58 . Hyponatremia (40\%), hypocalcemia (40\%), hypokalemia (30\%) and hypophosphatemia (30\%) were the main blood ionogram disturbances in malaria and dengue co-infection. The statistically significant disturbances in case of malaria and dengue co-infection were the absence of hypobicarbonatemia $(\mathrm{p}=0.036)$.
\end{abstract}

Conclusion: Malaria and dengue are responsible for significant morbidity and mortality in Burkina Faso. Although co-infection was rare in the study (1.14\%), it was associated with several blood ionogram disturbances. Evaluation and consideration of these disturbances during treatment would contribute to a better care of patients.

Keywords: pediatric university hospital, co-infection, dengue, blood ionogram, malaria

\section{Introduction}

Malaria and dengue are vector-borne diseases, which are public health problems worldwide and particularly in Burkina Faso. According to the World Health Organization (WHO), malaria was responsible for 228 million cases in 2018 with 405000 deaths, mostly among children under five years old (World Health Organization [WHO], 2019). Similarly, more than 390 million dengue virus infections occur worldwide each year, with 5.2 million cases reported to WHO in 2019 (WHO, 2021). Both diseases are found in common geographical areas, especially in the tropics and subtropics, and cases of co-infection are increasingly reported (Epelboin et al., 2012; Faruque et al., 2012; Mohapatra et al., 2012; Baba et al., 2013; Mendonça et al., 2015). In Burkina Faso, a survey on 264 children revealed 18 positive cases of dengue, including 10 cases associated with malaria (Ministry of Health, 2014). In 2017, out of 51 cases of dengue observed in the infectious disease department of the University Hospital Yalgado Ouedraogo at Ouagadougou, a co-infection with malaria was observed in 7 patients (Savadogo et al., 2017). These diseases are related to high morbidity, and in endemic areas, complications are responsible for a high number of deaths. The main complications arise from changes in certain haematological and biochemical characteristics, which can aggravate the clinical picture, but also from the immune response against the parasitized red blood cells or against the dengue virus. Thus, some biological complications may be 
life-threatening, such as severe thrombocytopenia, severe hepatitis, renal failure and potentially serious ionic disorders. These complications must be detected in the laboratory and treated appropriately to ensure patient survival.

In Burkina-Faso, few studies focused on the biological complications of malaria and dengue co-infection, particularly hydro-electrolytic disorders, making this study necessary. Thus, we studied the disturbances of blood ionogram in malaria and dengue co-infection at the pediatric university hospital Charles de Gaulle in order to allow a better care of the patients.

\section{Material and Methods}

The study was conducted in the medical analysis laboratory of the University Hospital Charles de Gaulle at Ouagadougou, Burkina Faso. It was a descriptive cross-sectional study, with retrospective data collection on a period of three (03) years, from January $1^{\text {st }}, 2017$ to December $31^{\text {st }}, 2019$. The population studied consisted of all patients (all ages) who had a thick blood drop/screen, dengue serology and biochemical examinations including blood ionogram. Sampling was systematic and exhaustive during the study.

The thick blood drop and the blood smear were performed on a slide from a capillary blood sample, stained with Giemsa and May-Grünwald Giemsa respectively. The thick drop was used to make a positive diagnosis of malaria; the blood smear was used to diagnose the species and to evaluate the parasite density. Dengue serology and biochemical assays were performed on venous blood samples collected in dry tubes, centrifuged at $3500 \mathrm{rpm}$ for 5 minutes with serum collection. The SD Bioline Dengue Duo kit (Standard Diagnostics) was used for the diagnosis of dengue and was considered as a probable case of dengue, any patient with a positive serology for NS1 antigen and/or immunoglobulin M. Blood ionogram parameters were represented by the serum determination of sodium, potassium, chlorine, calcium, magnesium, phosphate and proteins, using the Indiko Plus automaton (Thermo Fisher Scientific). The values obtained were interpreted according to the patient's age.

Data collected on a questionnaire were entered from Microsoft Office Excel 2013 software and analyzed with Epi-Info ${ }^{\mathrm{TM}}$ 7 software in its version 7.2.0. Univariate and bivariate regression analyses were performed to establish a statistical relationship between the variables. For univariate analysis, Chi-2 or Fischer tests were used and for bivariate analysis, measures of association such as Odds Ratio and $p$-value were performed. The significance level was $p<0.05$. Before the beginning of the study, a request for authorization to collect data was obtained from the management of the Pediatric University Hospital Charles de Gaulle. Data confidentiality was maintained throughout the study.

\section{Results}

A total of 1405 subjects were included in the study.

\subsection{Socio-Demographic Characteristics}

The mean age of the patients of the study was $9.93 \pm 10.26$ years with extremes from 1 day to 80 years. The age range of 0 to 15 years was the majority with 1109 patients, representing a frequency of $78.93 \%$. Male sex was the most represented with a sex ratio of 1.15. The sociodemographic characteristics are presented in Table 1.

Table 1. Distribution of patients according to socio-demographic characteristics

\begin{tabular}{llrr}
\hline Characteristic & & Number & Percentage (\%) \\
\hline Age & $\leq 15$ & 1109 & 78.93 \\
(years) & ] $15-30]$ & 138 & 9.82 \\
& ] $30-45]$ & 121 & 8.61 \\
& $>45$ & 37 & 2.63 \\
Gender & Male & 752 & 53.52 \\
& Female & 653 & 46.48 \\
\hline
\end{tabular}

\subsection{Prevalence of Malaria, Dengue and Co-infection}

Thick blood tests were positive in 102 patients, i.e. a prevalence of malaria of $7.26 \%$. The mean parasite density was 30 $163.93 \pm 67741.85$ trophozoites $/ \mu \mathrm{L}$, ranging from 110 to 520909 trophozoites $/ \mu \mathrm{L}$. Dengue serology was positive in 235 patients $(16.72 \%)$. Out of the 1405 patients, the frequency of malaria-dengue co-infection was $1.14 \%$ with $n=16$ (Figure $1)$. 


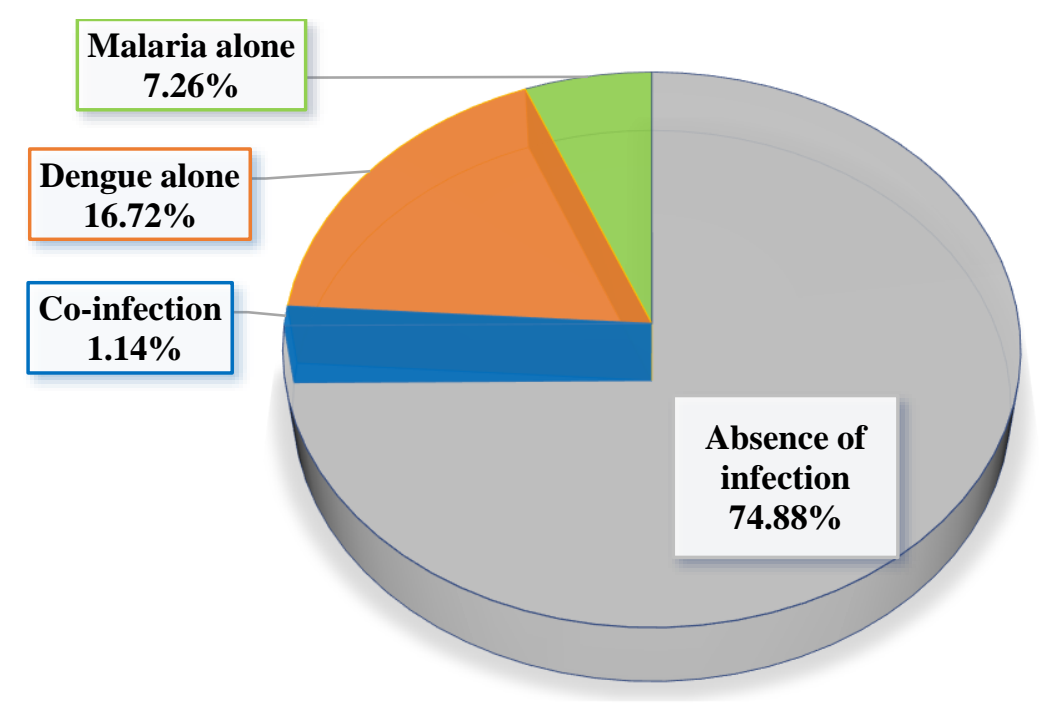

Figure 1. Frequency of malaria, dengue and co-infection

\subsection{Blood Ionogram Results}

\subsubsection{Mean Values of Blood Ionogram Parameters}

Table 2 presents the mean values obtained after the determination of the different parameters of the blood ionogram, according to the cases of co-infection, dengue or malaria.

Table 2. Mean values of blood ionogram parameters

\begin{tabular}{lrrr}
\hline Parameters & Co-infection & Dengue & Malaria \\
\hline Natremia $(\mathrm{mmol} / \mathrm{L})$ & $135.44 \pm 08.09$ & $134.71 \pm 05.57$ & $134.94 \pm 05.90$ \\
Kalemia $(\mathrm{mmol} / \mathrm{L})$ & $3.65 \pm 0.44$ & $4.35 \pm 0.80$ & $4.30 \pm 0.66$ \\
Calcemia $(\mathrm{mmol} / \mathrm{L})$ & $2.26 \pm 0.29$ & $2.35 \pm 0.35$ & $2.33 \pm 0.48$ \\
Chloremia $(\mathrm{mmol} / \mathrm{L})$ & $104.02 \pm 04.71$ & $99.87 \pm 11.74$ & $104.61 \pm 4.54$ \\
Phosphatemia $(\mathrm{mmol} / \mathrm{L})$ & $1.22 \pm 0.41$ & $1.55 \pm 0.46$ & $1.32 \pm 0.73$ \\
Magnesemia $(\mathrm{mmol} / \mathrm{L})$ & $0.80 \pm 0.40$ & $0.76 \pm 0.31$ & $0.71 \pm 0.24$ \\
Bicarbonate $(\mathrm{mmol} / \mathrm{L})$ & $23.89 \pm 3.48$ & $21.74 \pm 4.21$ & $19.69 \pm 4.26$ \\
Protidemia $(\mathrm{g} / \mathrm{L})$ & $72.64 \pm 11.65$ & $72.21 \pm 11.58$ & $66.4 \pm 11.75$ \\
\hline
\end{tabular}

\subsubsection{Frequency of disturbances}

The frequencies of blood ionogram disturbances are presented in Figure 2. In case of malaria-dengue co-infection, the main disturbances were hyponatremia (40\%), hypokalemia (30\%), hypocalcemia (40\%) and hypophosphatemia (30\%). 


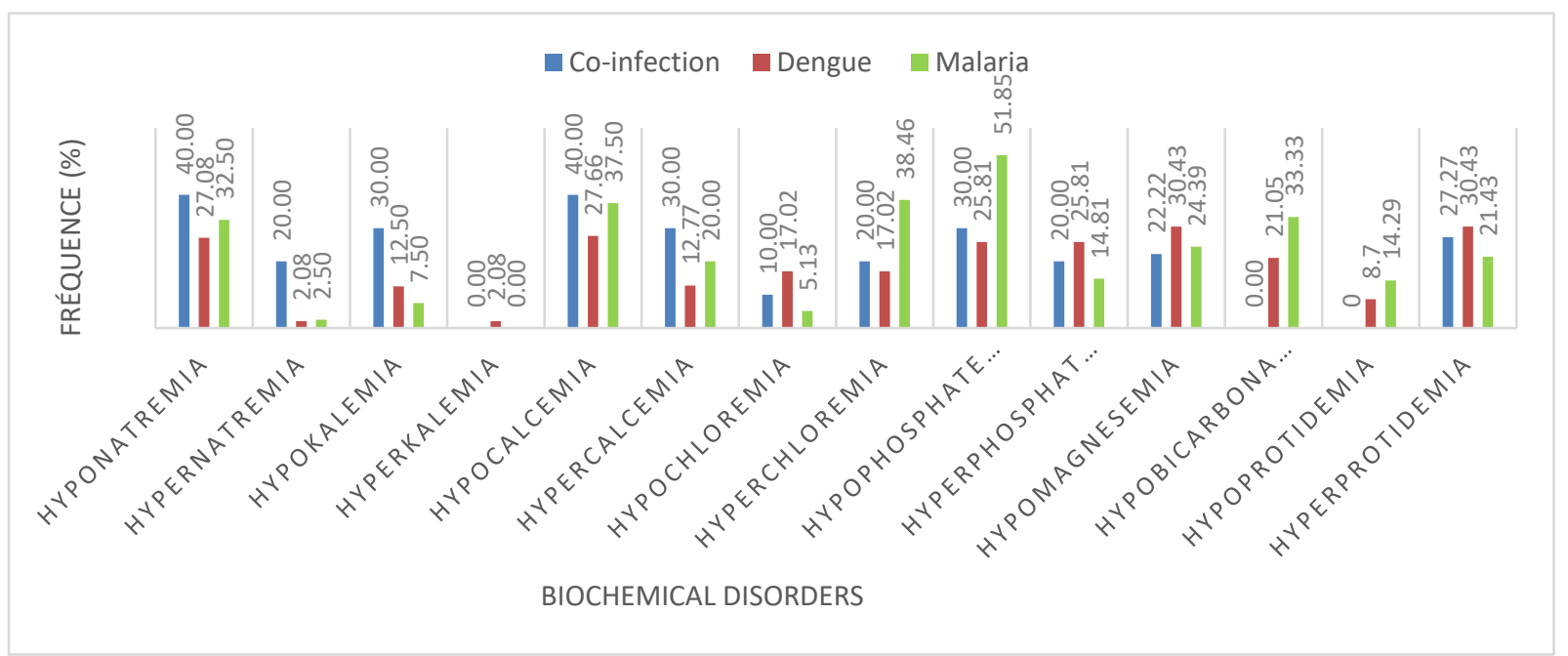

Figure 2. Frequency of blood ionogram disturbances

\subsubsection{Univariate Analysis}

In univariate analyses, the absence of hypobicarbonatemia $(\mathrm{p}=0.036)$ was significantly associated with malaria-dengue co-infection.

No abnormality was associated with the presence of dengue alone. Hypobicarbonatemia $(p=0.048)$, hyperchloremia $(\mathrm{p}=0.013)$ and hypophosphatemia $(\mathrm{p}=0.043)$ were present in malaria infection alone (Table 3$)$.

Table 3. Univariate analysis of blood ionogram disturbances between co-infection, dengue and malaria

\begin{tabular}{lccc}
\hline Disturbances & Co-infection & Dengue & Malaria \\
\hline Hypobicarbonatemia & $\mathbf{0 . 0 3 6}$ & 0.280 & $\mathbf{0 . 0 4 8}$ \\
Hyponatremia & 0.250 & 0.230 & 0.370 \\
Hyperchloremia & 0.350 & 0.060 & $\mathbf{0 . 0 1 3}$ \\
Hypercalcemia & 0.150 & 0.290 & 0.590 \\
Hypomagnesemia & 0.770 & 0.500 & 0.650 \\
Hypophosphatemia & 0.330 & 0.130 & $\mathbf{0 . 0 4 3}$ \\
Hypokalemia & 0.440 & 0.450 & 0.340 \\
Hyperprotidemia & 0.450 & 0.490 & 0.360 \\
\hline
\end{tabular}

\subsubsection{Bivariate Analysis}

Among biological disturbances, hypophosphatemia was less frequent in the co-infection group than in the malaria-only group $(\mathrm{OR}=0.24$; C195\%: 0.06-0.99; $\mathrm{p}$-value $=0.044)$. On the contrary, hypophosphatemia was more common in the co-infection group than in the dengue-only group $(\mathrm{OR}=7.04 ; \mathrm{CI} 95 \%$ : $1.52-32.63 ; \mathrm{p}$-value $=0.009)$.

\section{Discussion}

The aim of the present study was to investigate blood ionogram disturbances in malaria and dengue co-infection at the Pediatric University Hospital Charles de Gaulle. Despite the retrospective nature of the study with the fact that some clinical records were not complete, 1405 patients were included.

\subsection{Prevalence of Malaria And Dengue Co-Infection}

The prevalence of the co-infection in this study was 1.14\% (Figure 1), thus relatively low. In a study at Thaillande, on 194 dengue patients, no association with malaria has been found(Singhsilarak et al., 2006). Even if the phenomenon of co-infection seems to be rare in terms of frequency (Abdul-Ghani et al., 2021), it is often described in the literature. Thus, Im et al. identified in their study 39 cases of co-infection in 2762 patients, i.e. a frequency of $1.4 \%$; thus similar to our results (Im et al., 2020). The low frequency (1.14\%) could be explained by the fact that the study was limited to results recorded only in the laboratory. It could also be due to low recording of the results of thick blood drop in the registers of 
the parasitology department, but this remains to be confirmed with further studies. In addition, the study period did not coincide with dengue outbreak as the one which occurred in the city of Ouagadougou in October 2016; this epidemic being considered as the biggest of its history (Im et al., 2020; Lim et al., 2021). On the other hand, Mohapatra et al. reported a higher frequency of 7.4\% in 2012 in India (Mohapatra et al., 2012). In Burkina Faso, few data are available on malaria and dengue co-infection (Ministry of Health, 2014; Savadogo et al., 2017; Im et al., 2020), except for some doctoral theses which found co-infection frequencies of 20.4\% in 2017 (Ly, 2017) and 55.24\% in 2020 (Kane, 2020). The high prevalence of these studies may be explained by the methodology used, which included patients with only Ig $\mathrm{G}$ positivity in dengue cases. However, the presence of $\mathrm{Ig} G$ is most often a sign of old dengue and only titration could distinguish it from secondary dengue. All cases of positive Ig $\mathrm{G}$ alone were excluded from this present study, as titration was not available.

\subsection{Ionic Disorders}

Many studies focused on the biological aspects of malaria and dengue co-infection (Epelboin et al., 2012; Mohapatra et al., 2012; S. Ahmad et al., 2016; Shah \& Mehta, 2017; Kotepui \& Kotepui, 2019; A. Ahmad et al., 2019; Kotepui et al., 2020; Galani et al., 2021), but very few noted ionic disturbances. The present study highlighted numerous blood ionogram disturbances during the concomitant presence of malaria with dengue (Figure 2). However, the lack of evidence in the literature on the hydroelectrolytic disorders encountered during coinfection considerably limits discussion.

Hyponatremia was found in $40 \%$ of co-infected patients, in $27.08 \%$ of dengue cases alone and $32.5 \%$ of malaria cases. Regarding the results of dengue, Vachvanichsanong and McNeil found $46.7 \%$ of hyponatremia (Vachvanichsanong \& McNeil, 2015). Epelboin noted that $7.1 \%$ of patients co-infected with dengue and malaria had hyponatremia (Epelboin et al., 2012). In the literature, hyponatremia is the most common biochemical disturbance in dengue or malaria (Karnad et al., 2018; Rehman et al., 2020; Ng \& Cheong, 2021) ; but insufficiently documented in co-infection. However, on the one hand, this may be explained by an excess of water due to an increase in metabolism, an inappropriate transient secretion of antidiuretic hormone leading to an increased tubular reabsorption, an influx of sodium into the cells due to a dysfunction of the sodium-potassium pump, or a combination of these factors; and on the other hand by digestive losses of sodium (diarrhea, vomiting). Indeed, Kane noted $38 \%$ of patients presenting digestive signs as vomiting and diarrhea, responsible for extracellular dehydration leading to a significant hyponatremia (Kane, 2020).

Hypokalemia was present in $30 \%$ of patients with co-infection (Figure 2). It could be attributed to activation of the renin-angiotensin-aldosterone system resulting in loss of potassium in the urine. In contrast, hyperkalemia was absent in co-infection but only found in one patient $(2.08 \%)$ infected with dengue alone. These results are similar to those of Epelboin who recorded $1.5 \%$ in his study (Epelboin et al., 2012). Vachvanichsanong and McNeil reported an hyperkalemia of 5.95\% (Vachvanichsanong \& McNeil, 2015). This hyperkalemia could be explained on the one hand by the presence of metabolic acidosis and on the other hand by the altered renal function of some patients.

Hypocalcemia was present in $40 \%$ of patients with co-infection, $27.66 \%$ and $37.50 \%$ in isolated dengue and isolated malaria respectively (Figure 2). Hypocalcemia, although insufficiently reported in co-infection, is particularly well documented in dengue (Bunnag \& Kalayanarooj, 2011; Kapoor \& Singh, 2012; Constantine et al., 2014) and malaria (Ayoola et al., 2005; Aijazi et al., 2020). Hypocalcemia is observed in severe dengue and is thought to be associated with increased mortality. Hypercalcemia was found in $30 \%$ of co-infected patients, compared to $37.50 \%$ for malaria mono-infection and $27.66 \%$ for isolated dengue. Hypercalcemia could be explained by an excess of intravenous calcium intake or by the altered renal function of some of the patients. Calcium remains an important parameter to avoid cardiac complications.

Hyperchloremia was observed in $38.46 \%$ of malaria cases, $17.02 \%$ for dengue and $20 \%$ in case of co-infection (Figure 2). The results are similar to those of Syed et al. who demonstrated that serum chloride levels in dengue patients significantly increase (Syed et al., 2014). This observed hyperchloremia could be due to the extracellular dehydration of the patients due to vomiting, but also to the decrease of bicarbonates.

We reported hypophosphatemia in $30 \%, 25.81 \%$ and $51.85 \%$ of patients respectively with co-infection, dengue and malaria. Likewise, hypomagnesemia was found in $22.22 \%$ of co-infected patients, $24.39 \%$ with malaria and $30.43 \%$ of those suffering from dengue. Hypophosphatemia and hypomagnesemia could be explained by the presence of hypercalcemia and the renal dysfunction of some of our patients.

No cases of decreased bicarbonate ions or hypoprotidemia were noted during the study. However, $27.27 \%$ of cases of hyperprotidemia were present in case of co-infection, versus $21.43 \%$ for malaria and $30.43 \%$ for dengue. It could be explained by the presence of hemoconcentration due to digestive losses (vomiting, diarrhea).

\section{Conclusion}

Malaria and dengue are the two major stars of tropical infectious diseases and are endemic in Burkina Faso. Both diseases 
can be diagnosed in the same patient and the present study found a co-infection frequency of $1.14 \%$. It was found that the ionogram disturbances were dominated by hyponatremia, hypocalcemia, hypokalemia and hypophosphatemia. It seems appropriate that further studies be carried out to assess the correlation of these ionic disorders with the evolution of patients with malaria-dengue co-infection.

\section{Conflicts of interest}

The authors declare that they have no conflicts of interest.

\section{Acknowledgements}

We are grateful to all the staff of the laboratory of the Pediatric University Hospital Charles de Gaulle at Ouagadougou (Burkina Faso).

\section{References}

Abdul-Ghani, R., Mahdy, M. A. K., Alkubati, S., Al-Mikhlafy, A. A., Alhariri, A., Das, M., Dave, K., \& Gil-Cuesta, J. (2021). Malaria and dengue in Hodeidah city, Yemen: High proportion of febrile outpatients with dengue or malaria, but low proportion co-infected. PloS One, 16(6), e0253556. https://doi.org/10.1371/journal.pone.0253556

Ahmad, A., Khan, I. A., \& Raza, M. (2019). Clinico-hematological profile of children with Dengue and co-infection with Malaria: A hospital based study. https://doi.org/10.18203/2349-3291.ijcp20193687

Ahmad, S., Dhar, M., Mittal, G., Bhat, N. K., Shirazi, N., Kalra, V., Sati, H. C., \& Gupta, V. (2016). A comparative hospital-based observational study of mono- and co-infections of malaria, dengue virus and scrub typhus causing acute undifferentiated fever. European Journal of Clinical Microbiology \& Infectious Diseases: Official Publication of the European Society of Clinical Microbiology, 35(4), 705-711. https://doi.org/10.1007/s10096-016-2590-3

Aijazi, I., Chaudary, M., Adam Mukhtar, S. H., \& Abdulla Al Shama, F. M. (2020). Falciparum Malaria Presenting With Tetany: Endocrinopathies Associated With Falciparum Malaria. Journal of Ayub Medical College, Abbottabad: JAMC, 32(1), 136-138.

Ayoola, O. O., Fawole, O. I., \& Omotade, O. O. (2005). Calcium and phosphate levels in Nigerian children with malaria. Annals of Tropical Paediatrics, 25(4), 303-306. https://doi.org/10.1179/146532805X72467

Baba, M., Logue, C. H., Oderinde, B., Abdulmaleek, H., Williams, J., Lewis, J., Laws, T. R., Hewson, R., Marcello, A., \& D' Agaro, P. (2013). Evidence of arbovirus co-infection in suspected febrile malaria and typhoid patients in Nigeria. Journal of Infection in Developing Countries, 7(1), 51-59. https://doi.org/10.3855/jidc.2411

Bunnag, T., \& Kalayanarooj, S. (2011). Dengue shock syndrome at the emergency room of Queen Sirikit National Institute of Child Health, Bangkok, Thailand. Journal of the Medical Association of Thailand = Chotmaihet Thangphaet, 94 Suppl 3, S57-63.

Constantine, G. R., Rajapakse, S., Ranasinghe, P., Parththipan, B., Wijewickrama, A., \& Jayawardana, P. (2014). Hypocalcemia is associated with disease severity in patients with dengue. The Journal of Infection in Developing Countries, 8(09), 1205-1209. https://doi.org/10.3855/jidc.4974

Epelboin, L., Hanf, M., Dussart, P., Ouar-Epelboin, S., Djossou, F., Nacher, M., \& Carme, B. (2012). Is dengue and malaria co-infection more severe than single infections? A retrospective matched-pair study in French Guiana. Malaria Journal, 11, 142. https://doi.org/10.1186/1475-2875-11-142

Faruque, L. I., Zaman, R. U., Alamgir, A. S. M., Gurley, E. S., Haque, R., Rahman, M., \& Luby, S. P. (2012). Hospital-Based Prevalence of Malaria and Dengue in Febrile Patients in Bangladesh. The American Journal of Tropical Medicine and Hygiene, 86(1), 58-64. https://doi.org/10.4269/ajtmh.2012.11-0190

Galani, B. R. T., Mapouokam, D. W., Simo, F. B. N., Mohamadou, H., Chuisseu, P. D. D., Njintang, N. Y., \& Moundipa, P. F. (2021). Investigation of dengue-malaria coinfection among febrile patients consulting at Ngaoundere Regional Hospital, Cameroon. Journal of Medical Virology, 93(6), 3350-3361. https://doi.org/10.1002/jmv.26732

Im, J., Balasubramanian, R., Ouedraogo, M., Wandji Nana, L. R., Mogeni, O. D., Jeon, H. J., van Pomeren, T., Haselbeck, A., Lim, J. K., Prifti, K., Baker, S., Meyer, C. G., Kim, J. H., Clemens, J. D., Marks, F., \& Soura, A. B. (2020). The epidemiology of dengue outbreaks in 2016 and 2017 in Ouagadougou, Burkina Faso. Heliyon, 6(7), e04389. https://doi.org/10.1016/j.heliyon.2020.e04389

Kane, C. (2020). Perturbations des paramètres biochimiques au cours de la dengue au Centre Hospitalier Universitaire Pédiatrique Charles De Gaulle du ler Janvier 2017 Au 31 Décembre 2018 [Unpublished doctoral dissertation]. Université Joseph Ki-Zerbo, Ouagadougou, Burkina Faso. 
Kapoor, S., \& Singh, A. (2012). Hypocalcemic tetany: An infrequently recognized association with acute dengue infection. Indian Journal of Pediatrics, 79(12), 1673. https://doi.org/10.1007/s12098-012-0690-3

Karnad, D. R., Nor, M. B. M., Richards, G. A., Baker, T., Amin, P., \& Council of the World Federation of Societies of Intensive and Critical Care Medicine. (2018). Intensive care in severe malaria: Report from the task force on tropical diseases by the World Federation of Societies of Intensive and Critical Care Medicine. Journal of Critical Care, 43, 356-360. https://doi.org/10.1016/j.jcrc.2017.11.007

Kotepui, M., \& Kotepui, K. U. (2019). Prevalence and laboratory analysis of malaria and dengue co-infection: A systematic review and meta-analysis. BMC Public Health, 19(1), 1148. https://doi.org/10.1186/s12889-019-7488-4

Kotepui, M., Kotepui, K. U., Milanez, G. D. J., \& Masangkay, F. R. (2020). Prevalence of and risk factors for severe malaria caused by Plasmodium and dengue virus co-infection: A systematic review and meta-analysis. Infectious Diseases of Poverty, 9, 134. https://doi.org/10.1186/s40249-020-00741-z

Lim, J. K., Carabali, M., Edwards, T., Barro, A., Lee, J.-S., Dahourou, D., Lee, K. S., Nikiema, T., Shin, M. Y., Bonnet, E., Kagone, T., Kaba, L., Namkung, S., Somé, P.-A., Yang, J. S., Ridde, V., Yoon, I.-K., Alexander, N., \& Seydou, Y. (2021). Estimating the Force of Infection for Dengue Virus Using Repeated Serosurveys, Ouagadougou, Burkina Faso. Emerging Infectious Diseases, 27(1), 130-139. https://doi.org/10.3201/eid2701.191650

Ly, D. (2017). La co-infection de la dengue avec le paludisme dans la ville de Ouagadougou: Aspects épidémiologiques, cliniques, biologiques et évolutifs [Unpublished doctoral dissertation]. Université Joseph Ki-Zerbo, Ouagadougou, Burkina Faso.

Mendonça, V. R. R., Andrade, B. B., Souza, L. C. L., Magalhães, B. M. L., Mourão, M. P. G., Lacerda, M. V. G., \& Barral-Netto, M. (2015). Unravelling the patterns of host immune responses in Plasmodium vivax malaria and dengue co-infection. Malaria Journal, 14, 315. https://doi.org/10.1186/s12936-015-0835-8

Ministry of Health. (2014). Directives nationales de prise en charge des cas de dengue au Burkina Faso (p. 22). http://www.touteinfo.com/IMG/pdf/directives_pec_dengue_version_du_21_juillet.pdf

Mohapatra, M. K., Patra, P., \& Agrawala, R. (2012). Manifestation and outcome of concurrent malaria and dengue infection. Journal of Vector Borne Diseases, 49(4), 262-265.

Ng, W. W., \& Cheong, B. M. K. (2021). Dengue Encephalitis associated with symptomatic hyponatremia due to Syndrome of Inappropriate Antidiuretic Hormone Secretion. The Medical Journal of Malaysia, 76(2), 261-263.

Rehman, F. U., Omair, S. F., Memon, F., Amin, I., Rind, B. J., \& Aziz, S. (2020). Electrolyte Imbalance at Admission Does Not Predict the Length of Stay or Mortality in Dengue-Infected Patients. Cureus, 12(9), e10419. https://doi.org/10.7759/cureus.10419

Savadogo, M., Boushab, B. M., Fall-Malick, Z., Apoline, S., \& Sow, M. (2017). Aspects epidemiologiques et cliniques des cas de dengue observes dans le Service des Maladies Infectieuses du CHU Yalgado Ouedraogo de Ouagadougou. Annale de l'Université Ouaga 1 Pr Joseph KI-ZERBO - Série D.

Shah, P. D., \& Mehta, T. K. (2017). Evaluation of concurrent malaria and dengue infections among febrile patients. Indian Journal of Medical Microbiology, 35(3), 402-405. https://doi.org/10.4103/ijmm.IJMM_15_455

Singhsilarak, T., Phongtananant, S., Jenjittikul, M., Watt, G., Tangpakdee, N., Popak, N., Chalermrut, K., \& Looareesuwan, S. (2006). Possible acute coinfections in Thai malaria patients. The Southeast Asian Journal of Tropical Medicine and Public Health, 37(1), 1-4.

Syed, S., Mahmood, Z., Riaz, M., Latif, S., Majeed, N., \& Rashid, A. (2014). Elemental profile of blood serum of dengue fever patients from Faisalabad, Pakistan. Int J Biol Sci, 6, 34-37.

Vachvanichsanong, P., \& McNeil, E. (2015). Electrolyte disturbance and kidney dysfunction in dengue viral infection. The Southeast Asian Journal of Tropical Medicine and Public Health, 46 Suppl 1, 108-117.

World Health Organization (WHO). (2019). World malaria report 2019. https://www.who.int/publications-detail-redirect/9789241565721

World Health Organization (WHO). (2021). Dengue et dengue sévère. https://www.who.int/fr/news-room/fact-sheets/detail/dengue-and-severe-dengue

\section{Copyrights}

Copyright for this article is retained by the author(s), with first publication rights granted to the journal.

This is an open-access article distributed under the terms and conditions of the Creative Commons Attribution license (http://creativecommons.org/licenses/by/4.0/). 